\title{
Luminescence Lifetime Distributions Analysis in Heterogeneous Systems by the Use of Excel's Solver
}

\author{
Tiago J. F. Branco, Ana M. Botelho do Rego, Isabel Ferreira Machado, and \\ Luis F. Vieira Ferreira* \\ Centro de Química-Física Molecular, Complexo Interdisciplinar, Instituto Superior Técnico, Av. Rovisco Pais, \\ 1049-001 Lisboa, Portugal
}

Received: April 18, 2005; In Final Form: June 15, 2005

\begin{abstract}
A detailed study of the luminescence decay curves of pyrene included within $p$-tert-butylcalix[4]arene cavities and benzophenone into silicalite channels is reported. A new methodology for a lifetime distribution analysis of the decay curve of probes onto heterogeneous surfaces is presented, which allows for asymmetric distributions and uses Voigt profiles (Gaussian and Lorentzian mixture) instead of pure Gaussian or Lorentzian distributions. Our approach uses a very simple and widely available tool for fitting, the Microsoft Excel Solver. In the case of the pyrene/tert-butylcalix[4]arene sample, the room temperature luminescence detected in the microsecond time scale was not only the phosphorescence of pyrene but also monomer delayed fluorescence, crystal phosphorescence, and excimer delayed fluorescence. In the benzophenone/silicalite case, three emissive forms of benzophenone could be assigned, one of benzophenone included into the silicalite circular zigzag channels, another for emplacement into the elliptical straight channels and finally when benzophenone is placed at the crossing points of those silicalite channels, where smaller spatial restrictions for benzophenone exist.
\end{abstract}

\section{Introduction}

To gain more information regarding photochemical processes in heterogeneous environments (opaque or powdered samples) a considerable scientific interest was made in spectroscopic and kinetic studies of organic molecules adsorbed onto different solid substrates with relevance for the use of time-resolved methodologies, i.e., time-resolved luminescence and transient absorption spectroscopies. ${ }^{1-5}$

The solid support can deeply affect or even control the photochemistry of an adsorbed probe. Good examples of this are photochemical studies of polycyclic aromatic hydrocarbons, ketones, and dyes, among others, on solid powdered substrates, such as hydrophobic or hydrophilic zeolites, ${ }^{4}$ silicas with different porosities, ${ }^{\text {a a }}$ aluminas, and silica-aluminas. ${ }^{5}$ Supramolecular studies of probes within cavities of cyclodextrins ${ }^{6}$ or calixarenes $^{7,8}$ should also be mentioned, among other supramolecular studies.

Reflection spectroscopies for the study of highly scattering samples require setup modifications both for ground-state and excited state studies. In the former case integrating spheres are used to obtain ground state absorption spectra of probes adsorbed onto powdered solid supports. With the use of suitable "white" and "black" standards for calibrating purposes, accurate values of the diffuse reflectance $R$ can be obtained as a function of the wavelength, and from those, the remission function $F(R)$ can be calculated for optically thick samples by the use of KubelkaMunk function. ${ }^{9,10}$ In this way, a direct correlation of $F(R)$ with the probe concentration of the sample can be obtained. Also the formation of aggregates of the probe onto a specific surface can be detected and studied ${ }^{10}$ using this ground-state technique.

Fluorescence and phosphorescence studies of molecules adsorbed on solids are different from the ones of homogeneous

* To whom correspondence should be addressed. E-mail: LuisFilipeVF@ist.utl.pt. Telephone: $351-21 \quad 841 \quad 92 \quad 52 . \quad$ Fax: 351-21 8464455. solutions (transparent samples) in several aspects, namely the necessity for the use of front-face geometry (also called reflection geometry), because the samples are opaque.

Today's availability of intensified charged-coupled devices (ICCDs) as detectors combined with the use of delay units and also laser excitation sources, allows one to perform timeresolved spectroscopic studies, provided the suitable time gate widths are selected. In this way the spectroscopist can separate prompt emissions (fluorescence) occurring immediately after the laser pulse, from delayed emissions (phosphorescence and delayed fluorescence, usually in a microsecond to second time scale), by controlling delays and gate-widths of the detection systems. $^{11}$

In studies of the relaxation of electronic excited states of probes adsorbed on solids, following photon absorption, this heterogeneous nature of the adsorption sites of solid powdered samples usually implies complex kinetics. ${ }^{12}$

A common starting point in the approaches for studying such systems is the use of sums of two or three exponentials to analyze an experimental decay curve (transient emission or transient absorption data). However this approach has no physical significance because the emitting probe does not exist in two or three emissive forms; instead usually it is one species that is emitting in different environments, and a distribution of sites of adsorption should be considered. Therefore, the decay analysis requires a very detailed knowledge of the specific physical and chemical interactions of the probe with the solid support sites.

More developed formalisms are used for analyzing decays of probes adsorbed on heterogeneous solid supports. They all assume that a distribution $\alpha(X)$ of emissive species exists and that the decay curve, $I(t)$ is given by

$$
I(t)=\int \alpha(X) \exp (-k t) d X
$$


where $k=1 / \tau$ is the decay rate and X may be either the lifetime $\tau$ ( $\tau$ space) or $\ln (\tau)[\ln (\tau)$ (or $\ln (k))$ space] and the integral extends from 0 to $+\infty$ in the first case and from $-\infty$ to $+\infty$ in the second one. The problem of recovering the distribution of lifetimes, $\alpha(X)$, from the decay curve is an inverse problem like many others in physical science, and its ill-conditioned nature is well-known and largely discussed in the literature. ${ }^{12}$ In practice, the integration is numerically solved by the use of a sum of terms, i.e., the integral in eq 1 is replaced by eq 2 where $\tau$ or $\ln (\tau)$ are equally spaced:

$$
I(t)=\sum \alpha(X) \exp (-k t)
$$

Theoretical arguments favor the use of the $\ln (\tau)$ space. As an example, in the dispersive kinetic analysis (DKA), ${ }^{13}, 14$ Gaussian uni- or multimodal distribution of the free energy is considered to take into account the heterogeneity of a system. The free energy is therefore described by a Gaussian distribution characterized through an average free energy $\overline{\Delta G^{0}}$ and a width parameter $\gamma\left(\Delta G^{0}=\overline{\Delta G^{0}}-\gamma x R T\right)$. Since $\Delta G^{0}=-R T \ln (k)=$ $-R T \ln (1 / \tau)=R T \ln (\tau)$ the space $\ln (\tau)$ is, in principle, more adequate than the space $\tau$. In the literature, examples of works in both spaces are found. ${ }^{13-16}$ When $\gamma=0$, the kinetics is no longer dispersive and the decays are monoexponential. The observed transient decay in this model is considered to be a summation of the contributions of each microscopic species in slightly different environments, instead of an integral.

In both spaces, distribution $\alpha$ can be unconditioned ${ }^{12}$ leading to a large number of parameters to be fitted (typically between 100 and 200) and many efforts were done to find a robust procedure to recover them. Another option is to assume that $\alpha$ obeys a given distribution uni- or multimodal. Gaussian, ${ }^{13}$ Poissonian, ${ }^{12}$ Lorentzian, ${ }^{15}$ and Levy ${ }^{14}$ distributions have been used in the literature.

Concerning the fitting criterion, there are basically two criteria: minimization of chi-square (inappropriately referred in the literature as exponential series method, ESM, ${ }^{17}$ because in all cases exponential series are used) and maximization of entropy (referred in the literature as $\mathrm{MEM}^{12}$ ). Usually multimodal distributions are obtained but rarely with more than three maxima. Given the nature of the problem, the option by a sum of conditioned distributions, leading to a much lower number of parameters to be fitted, seems a sounder one. The analysis of the decay of alloxazines and isoalloxazines on cellulose by Sikorsky et al. ${ }^{6}$ and of other systems by Kerry Thomas ${ }^{5}$ are among the works where the lifetime distribution analysis gives important insight into photophysics of the system.

In this paper, we will use a lifetime distribution analysis in space $\ln (\tau)$ but allowing for asymmetric distributions and Voigt profiles (Gaussian and Lorentzian mixture) instead of pure Gaussian distributions. Our approach gives a large flexibility to the model and innovates in the tool used for fitting, which is the Microsoft Excel available to all the PC or Macintosh users, in this way avoiding the commercially available softwares for MEM or ESM. Simulated decays with and without added noise are used to test the robustness of the method here proposed.

Finally, the method is applied to the analysis of the luminescence decay curves of pyrene included within p-tertbutylcalix[4]arene cavities and also benzophenone into silicalite channels. The time range under use here is high enough to neglect the distortion effect of the instrument response function at early times of the decay curve.

\section{Model}

A model of distribution based on the Gaussian model of Albery et al. ${ }^{13}$ applied by Krasnansky et al. ${ }^{16}$ to the simulation of heterogeneous systems was used. In this model, it is assumed that the lifetimes distribution of an excited probe adsorbed on a heterogeneous, porous substrate is a consequence of a distribution of $\Delta G^{0}$ of activation for the probe adsorption on the substrate around a mean value $\overline{\Delta G^{0}}$

$$
\Delta G^{0}=\overline{\Delta G^{0}}-\gamma x R T
$$

or, in terms of first rate constant, $k$

$$
\ln (k)=\ln (\bar{k})-\gamma x \Longrightarrow k=(\bar{k}) \exp (-\gamma x)
$$

where $\gamma$ is a measure of the distribution width (half full width at maximum $\times \exp (-1)$ ). In most of the preceding works, the distribution of species is assumed to be purely Gaussian $\left(\propto \exp \left(-x^{2}\right)\right)$ or Poissonian and symmetrical. The improvement in this work is to admit that the distribution function is a sum of symmetrical or asymmetrical Voigt profiles-GaussianLorentzian products-defined in eq 5 :

$$
\begin{aligned}
& \alpha\left(\ln \left(k_{j}\right)\right)= \\
& \sum_{i} \frac{\alpha_{\max , i} \exp \left(-\left(1-m_{i}\right)\left(\ln \left(k_{j}\right)-\ln \left(k_{i}\right)\right)^{2} \ln (2) /\left(a_{i} L_{i} / 2\right)^{2}\right)}{1+m_{i}\left(\ln \left(k_{j}\right)-\ln \left(k_{i}\right)\right)^{2} /\left(a_{i} L_{i} / 2\right)^{2}}
\end{aligned}
$$

Here $\alpha\left(\ln \left(k_{j}\right)\right)$ is the relative weight of $k_{j}$ in the total distribution, $\bar{k}_{i}$ is the position of the maximum of profile $i, \alpha_{\max , i}$ is its maximum, $L_{i}$ is its full width at half-maximum (fwhm), $m_{i}$ is the Lorentzian weight in the profile $i\left(m_{i}=1\right.$ corresponds to a pure Lorentzian and $m_{i}=0$ corresponds to a pure Gaussian), and $a_{i}$ is the asymmetry factor taking the value 1 for $k_{j} \geq \bar{k}_{i}$ and a value $\geq 0$ for $k_{j}<\vec{k}_{i}$.

The decay curve will, then, be given by

$$
I(t)=\sum_{j} \alpha\left(\ln \left(k_{j}\right)\right) \exp \left(-k_{j} t\right)
$$

or, in terms of normalized intensity:

$$
\frac{I(t)}{I(0)}=\frac{\sum_{j} \alpha\left(\ln \left(k_{j}\right)\right) \exp \left(-k_{j} t\right)}{\sum_{j} \alpha\left(\ln \left(k_{j}\right)\right)}
$$

The goal is to extract from experimental data, $I(t)$, the distribution $\alpha\left(\ln \left(k_{j}\right)\right)$.

\section{Methodology}

The method uses an Excel worksheet and its Solver tool, which has the advantage of being available in every Macintosh or PC and familiar to a large number of members of the international scientific community. However, any other software, even noncommercial, including a tool similar to Excel's Solver may be used. Four criteria for optimization of parameters were tested: least squares, $\left(\sum_{i}\left(I_{i}(t) \exp -I_{i}(t)_{\mathrm{fit}}\right)^{2}\right), \chi^{2}\left(\sum_{i}\left(I_{i}(t) \exp -\right.\right.$ $\left.\left.I_{i}(t)_{\mathrm{fit}}\right)^{2} / I_{i}(t) \exp \right)$, modified $\chi^{2}\left(\sum_{i}\left(I_{i}(t)_{\exp }-I_{i}(t)_{\mathrm{fit}}\right)^{2} /\left(I_{i}(t) \exp \right)^{2}\right)$, and mean value between maximum and minimum absolute deviation $\left(\left(\max \left(\operatorname{abs}\left(I_{i}(t) \exp -I_{i}(t)_{\mathrm{fit}}\right)\right)+\min \left(\operatorname{abs}\left(I_{i}(t) \exp -\right.\right.\right.\right.$ $\left.\left.\left.\left.I_{i}(t)_{\mathrm{fit}}\right)\right)\right) / 2\right)$. 
TABLE 1: Parameters Used for Simulation of Distributions Generating Test Decay Curves Compared to the Fitted Ones (without Any Added Noise)

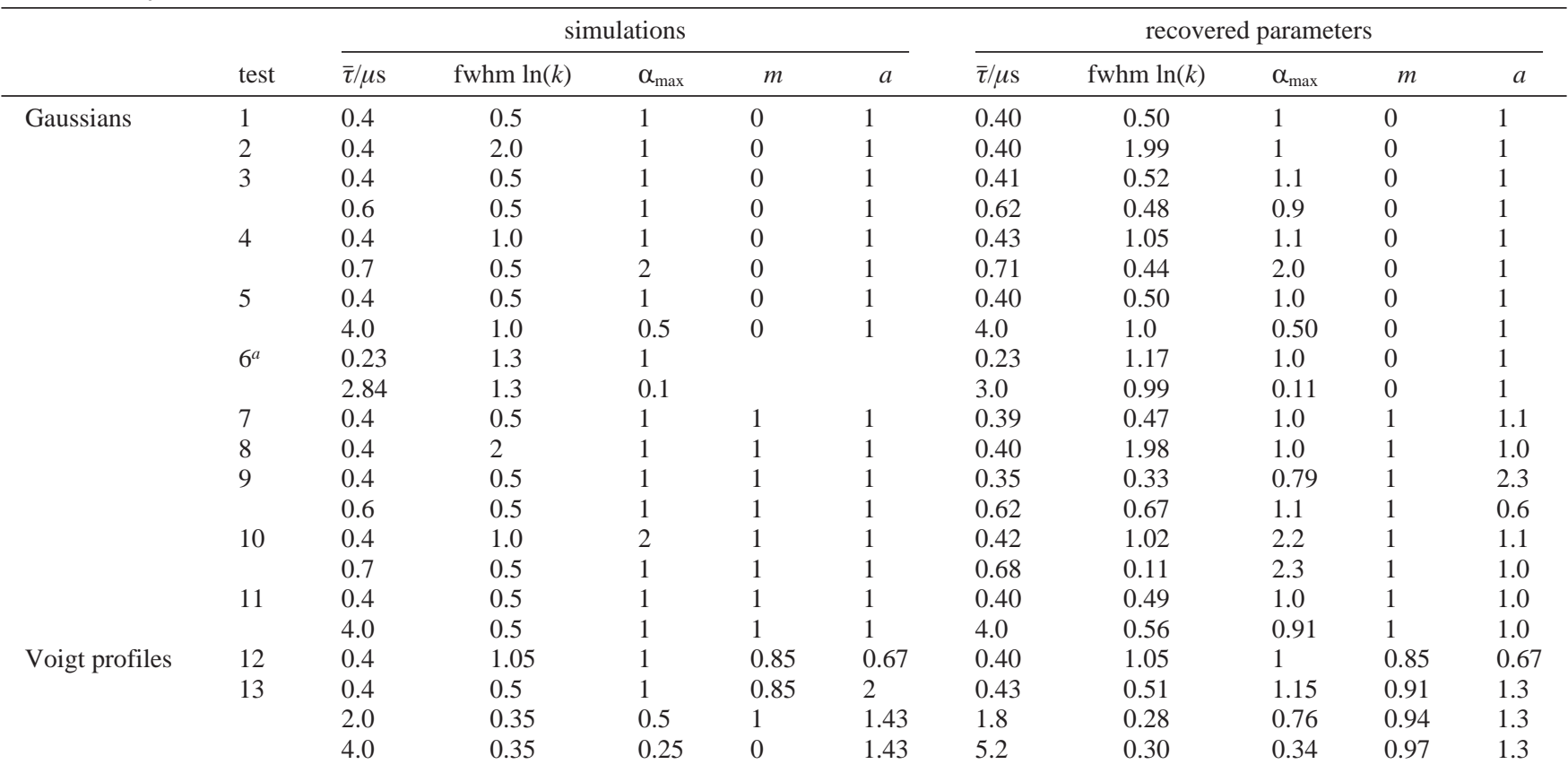

${ }^{a}$ Distribution was trapezoidal (see Figure 1).

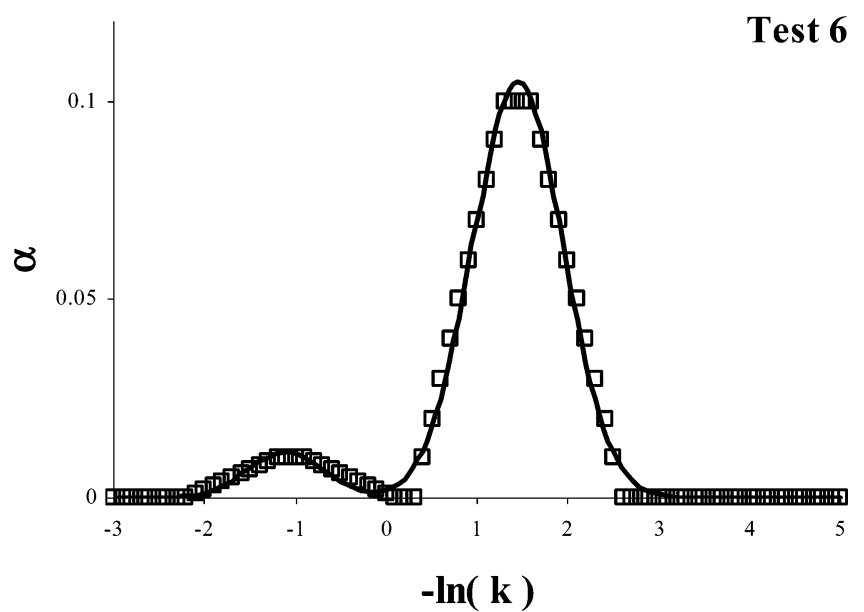

Figure 1. Test 6: Simulated bitrapezoidal distribution (squares) and fitted Voigt profiles (full line).

The ones which have shown better performance for all the tests were the least squares and the modified $\chi^{2}$ parameters, depending on the total range of data.

The first and last sets of columns of the worksheet are prepared for a maximum of three Voigt profiles (the extension for a larger number of components is straightforward, however, in the usually studied systems, three is enough). Its full description is presented in Appendix 1 and the optimization protocol in Appendix 2.

Tests. To test the robustness of the method, a few distributions were simulated and blindly tested. Table 1 shows the parameters of distributions used for simulating decay curves without noise as well as the parameters of the fitted curves.

For decay curves without noise, despite the ill-conditioned nature of the problem, the success in recovering distributions from decay curves is very high, especially for Gaussian distributions as can be seen in Table 1 data. Even for the trapezoidal distribution, a surprisingly good result was obtained. In Figure 1, the bitrapezoidal distribution fitted with Voigt profiles distributions is shown.
The Lorentzian distributions are more problematic because of their extended tails which, for parameters similar to the ones used in Gaussian distributions, require a larger range of $\ln (k)$. Therefore, in some cases, a larger number of columns in the worksheet or a larger value for the step $(\Delta[\ln (k)])$ have to be used. If one pays attention to these details, good fittings can be obtained even for the most unfavorable tests.

When the recovered parameters are different from the ones used in the simulation as in the case of test number 10 , the obtained distribution is still very close to the simulated one.

The final test for the method was provided by simulated decay curves where some noise was added. The random function of Excel was used to simulate the noise: to the calculated intensity, the function (rand ()$-0.5) F$ was added. Factor $F$ was chosen so that the noise was of the order of magnitude of the experimental one or even larger. Figure 2 displays the simulated decay curves with added noise.

Table 2 presents all parameters used for simulations and the recovered parameters which show the obtained results.

Obviously, there are some limitations namely in the resolving power: in test number 21 , corresponding to close distributions with the same relative weight, the fitting procedure recovers a single distribution that fits reasonably well the sum of the two distributions.

In test number 24, the most unfavorable case, the sum of two Voigt profiles with different fwhm, different relative intensities, and different asymmetries is used. As we can see, the fit is not perfect (it has been the worst result obtained), but even so, it gives a recovered distribution very close to the simulated one.

Following the fitting procedure described in detail in Appendix 2, all the tests converge for a unique and sound solution, provided the experimental decay curve is only moderately noisy.

\section{Experimental Section}

Materials. Benzophenone (Koch Light, purissimum) and p-tert-butylcalix[4]arene (Aldrich) were used without any 

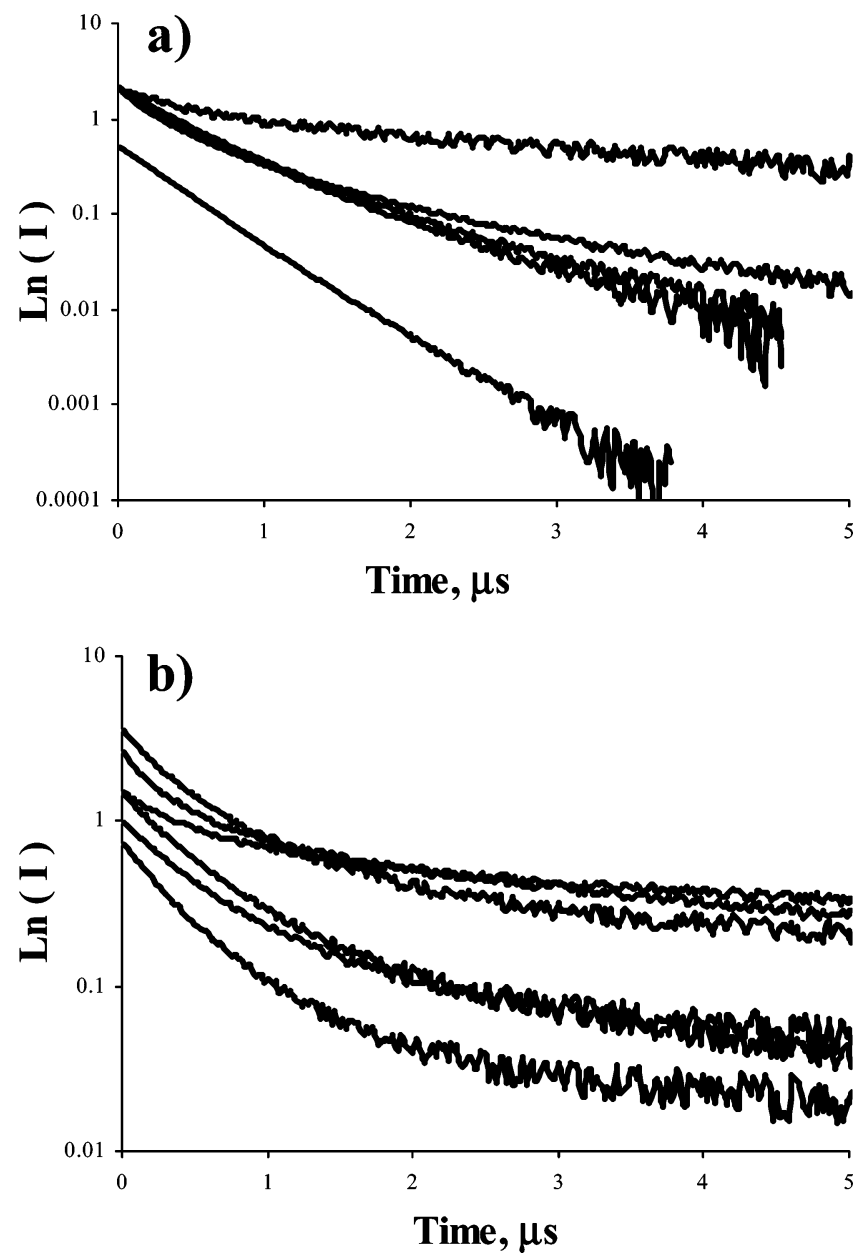

Figure 2. Simulated decay curves with added noise: (a) Gaussian distributions: from bottom to top: test 14 to test 18; (b) Lorentzian distributions: from bottom to top: test 19 to test 24 .

purification. Chloroform, isooctane, and ethanol (Merck, Uvasol grade) were also used as received. Silicalite was from Union Carbide.

Sample Preparation. The solid powdered samples pyrene/ calixarene (molar ratios 1:25, 1:50) used in this work were prepared using the solvent evaporation method. This method consists of the addition of a solution containing the probe to a saturated solution of the calixarene $\left(\sim 10^{-2} \mathrm{M}\right)$, both in chloroform. The resulting mixture was magnetically stirred for at least $24 \mathrm{~h}$ and then allowed to evaporate in a fume cupboard. The final solvent removal was performed overnight in an acrylic chamber with an electrically heated shelf (Heto, Model FD 1.0110) with temperature control $\left(30 \pm 1^{\circ} \mathrm{C}\right)$ and under moderate vacuum at a pressure of ca. $10^{-3}$ Torr. The evaluation of the existence of final traces of solvent was monitored by the use of FTIR spectra.

For silicalite samples, benzophenone selective adsorption into the silicalite channels was achieved using isooctane, ${ }^{1}$ whose molecular dimensions prevent this solvent to penetrate into the host channels. Following the initial solvent evaporation, samples were washed three times with isooctane for complete removal of the nonincluded ketone and dried again as described.

Laser Induced Luminescence Setup. A schematic diagram of the LIL system is presented in ref 1a. For the emission spectra shown, the third harmonic of a Nd:YAG laser $(355 \mathrm{~nm}$, ca. 6 ns fwhm) from B. M. Industries (Thomson-CSF, model Saga $12-10)$, in the diffuse reflectance mode were employed. The light arising from the solid powdered samples after excitation
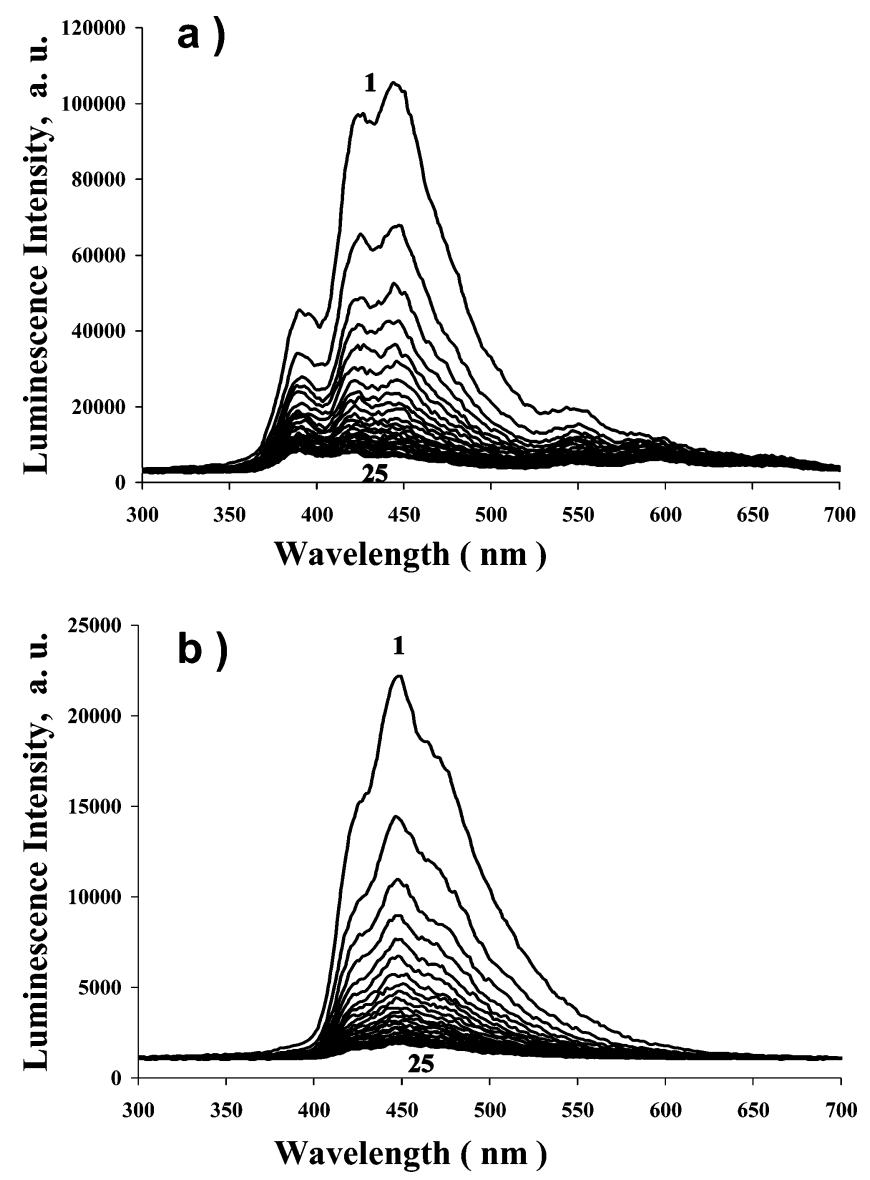

Figure 3. Time-resolved luminescence spectra (in a microsecond time scale) of pyrene adsorbed on $p$-tert-butylcalix[4]arene (a) and of pyrene crystals (b), excited at $337 \mathrm{~nm}$. Curve 1 was obtained $10 \mu$ s after the laser pulse. Curves $1-25$ are separated by $10 \mu$ s steps.

by the laser pulse was collected by a collimating beam probe coupled to an optical fiber (fused silica) and detected by a gated intensified charge coupled device (Andor ICCD detector, based on the Hamamatsu S5769-0907). The ICCD was coupled to a fixed imaging compact spectrograph (Oriel, model FICS 77441). The system could be used either by capturing all light emitted by the sample or in a time-resolved mode by using a delay box (Stanford Research Systems, model D6535). The ICCD has high-speed gating electronics $(2.2 \mathrm{~ns})$ and intensifier and covers the 200-900 $\mathrm{nm}$ wavelength range. Time-resolved emission spectra (and absorption) were available in the nanosecond to second time range both in transmission or diffuse reflectance modes. ${ }^{1}$ For the laser-induced luminescence experiments, a $\mathrm{N}_{2}$ laser (PTI model 2000, ca. $600 \mathrm{ps}$ fwhm, $\sim 1.0 \mathrm{~mJ} / \mathrm{pulse}$ ) was also used.

\section{Results and Discussion}

Room-Temperature Laser Induced Luminescence for Pyrene/p-tert-Butylcalix[4]arene. Figure 3a shows the roomtemperature luminescence spectra of a 1:50 mol:mol inclusion complex of pyrene and calix[4]arene while Figure 3b presents similar data obtained for pyrene microcrystals. In this case, as an excitation source we used the short pulse of a nitrogen laser at $337 \mathrm{~nm}$ (600 ps halfwidth) quite suitable to pyrene timeresolved emission studies in the microsecond time range, due to its short duration. All time-resolved spectra presented in Figure 3 were obtained with air equilibrated samples.

For pyrene within the calix host, spectra were also recorded for argon purged samples and were identical in spectral and 
TABLE 2: Parameters Used for Simulation of Distributions Generating Decay Curves in Figure 2 Compared to the Fitted Ones

\begin{tabular}{|c|c|c|c|c|c|c|c|c|c|c|c|}
\hline & \multirow[b]{2}{*}{ test } & \multicolumn{5}{|c|}{ simulations } & \multicolumn{5}{|c|}{ recovered parameters } \\
\hline & & $\bar{\tau} / \mu \mathrm{s}$ & fwhm & $\alpha_{\max }$ & $m$ & $a$ & $\bar{\tau} / \mu \mathrm{s}$ & fwhm & $\alpha_{\max }$ & $m$ & $a$ \\
\hline \multirow[t]{8}{*}{ Gaussians } & 14 & 0.4 & 0.5 & 1 & 0 & 1 & 0.39 & 0.48 & 1.0 & 0 & 1.1 \\
\hline & 15 & 0.4 & 2.0 & 1 & 0 & 1 & 0.41 & 2.1 & 1.0 & 0 & 0.96 \\
\hline & 16 & 0.4 & 0.5 & 1 & 0 & 1 & 0.44 & 0.72 & 1.4 & 0 & 1.6 \\
\hline & & 0.6 & 0.5 & 1 & 0 & 1 & 0.52 & 1.6 & 0.51 & 0 & 0.74 \\
\hline & 17 & 0.4 & 0.5 & 2 & 0 & 1 & 0.41 & 0.61 & 1.8 & 0 & 1 \\
\hline & & 0.7 & 1.0 & 1 & 0 & 1 & 0.70 & 1.0 & 0.90 & 0 & 1 \\
\hline & 18 & 0.4 & 0.5 & 1 & 0 & 1 & 0.40 & 0.62 & 1.5 & 0 & 1 \\
\hline & & 4.0 & 1.0 & 0.5 & 0 & 1 & 3.8 & 0.86 & 1.2 & 0 & 1 \\
\hline \multirow[t]{8}{*}{ Lorentzians } & 19 & 0.4 & 0.5 & 1 & 1 & 1 & 0.40 & 0.62 & 0.86 & 1.0 & 0.93 \\
\hline & 20 & 0.4 & 2 & 1 & 1 & 1 & 0.40 & 1.9 & 1.0 & 1 & 1.0 \\
\hline & 21 & 0.4 & 0.5 & 1 & 1 & 1 & 0.51 & 0.63 & 1.7 & 1.0 & 0.88 \\
\hline & & 0.6 & 0.5 & 1 & 1 & 1 & & & & & \\
\hline & 22 & 0.4 & 1.0 & 2 & 1 & 1 & 0.43 & 1.13 & 2.0 & 0.92 & 1 \\
\hline & & 0.7 & 0.5 & 1 & 1 & 1 & 0.58 & 0.65 & 0.55 & 1.0 & 1 \\
\hline & 23 & 0.4 & 0.5 & 1 & 1 & 1 & 0.39 & 0.50 & 1 & 1 & 1 \\
\hline & & 4.0 & 0.5 & 1 & 1 & 1 & 4.1 & 0.51 & 1 & 1.0 & 1 \\
\hline \multirow[t]{2}{*}{ Voigt profiles } & 24 & 0.4 & 0.5 & 1 & 0.85 & 2 & 0.36 & 0.58 & 0.80 & 0 & 2.63 \\
\hline & & 4.0 & 0.25 & 0.25 & 0 & 1.43 & 4.638 & 0.36 & 0.28 & 0 & 1.24 \\
\hline
\end{tabular}

intensity terms, within experimental error. For pyrene crystals a small increase of magnitude was found in the luminescence intensity with oxygen removal, but no difference could be detected in the luminescence decay curves.

Calixarenes are important macrocyclic phenol-formaldehyde polycondensates with hydrophobic bowl-shaped cavities. ${ }^{18,19}$ The ability of calixarenes and calixarene derivatives to form inclusion complexes, accommodating guest molecules in their intramolecular cavities, greatly depends on the size and geometry of guest molecule but also on the host cavity. ${ }^{7,8,11,18,19}$ Calixarenes can be extremely useful hosts for use in environmental chemistry studies due to their ability to selectively bind, separate, and sense neutral organic molecules (and ions) of contaminants, depending not only on the different sizes of its nanocavities but also on different substituents in the upper or lower rims. ${ }^{19}$

The time-resolved fluorescence spectrum of the 1:50 mol: mol pyrene/calix[4]arene sample in the nanosecond time range (data not shown) is very simple, exhibiting monomer emission peaking at $396 \mathrm{~nm}$ and also excimer emission peaking at about $470 \mathrm{~nm}$, as reported for pyrene on other solid powdered surfaces. ${ }^{20,21}$ The only notable fact is that the I/III monomer band ratio evidences a nonpolar environment $(\mathrm{I} / \mathrm{III}=0.80)$, pointing to the inclusion of pyrene in the nonpolar cavity of the calix[4]arene. The lifetimes for monomer and excimer emission in the pyrene/calix[4]arene sample in air equilibrated samples were about 220 and 40 ns, respectively.

The time-resolved spectra in the microsecond time scale (shown in Figure 3a) is much more complex and at the same time much more interesting. It shows several species emitting simultaneously: peaking at $396 \mathrm{~nm}$ is an emission coincident with the pyrene monomer fluorescence but which is now in the microsecond time scale, which we assign to delayed monomer emission of included pyrene. This type of delayed emission was reported before for pyrene in acetonitrile and in the presence of heavy atoms which induce room-temperature phosphorescence (RTP) and delayed fluorescence. ${ }^{22,23}$

The second important luminescence arises from pyrene crystals, which in fact comprise the pyrene crystal emission and also excimer delayed emission, the first one with a vibronic structure peaking at about 424 and $447 \mathrm{~nm}$ and the second one appearing as a small shoulder at about $479 \mathrm{~nm}$, which is as broad as it is in the excimer fluorescence case. The roomtemperature emission from pyrene microcrystals is shown for comparison in Figure 3b. These pyrene crystal emissions are in accordance with literature where evidence for pyrene microcrystallites was found on silica surfaces, ${ }^{24,21 \mathrm{~b}}$ agar solid matrix, ${ }^{25}$ and porous crystalline cellulose. ${ }^{26}$ The vibronic shoulder at $424 \mathrm{~nm}$ decreases in the case of the pure pyrene crystals when compared with pyrene onto calix[4]arene due to reabsorption of the luminescence which is larger in the former case.

Finally there is also room-temperature phosphorescence (RTP) from pyrene (air equilibrated sample), peaking at about $550 \mathrm{~nm}$, also possible to identify by comparison with pyrene phosphorescence studies either at low temperatures, ${ }^{21 \mathrm{~b}}$ or induced by an external heavy atom effect. ${ }^{22,23}$

What we have here is the calixarene protection from the oxygen existing in the air because of the inclusion complex formation and at the same time some pyrene also remained outside the calixarene cavities forming "pools" of pyrene microcrystals. A similar behavior was reported by us for benzophenone included within calix[n]arenes $(n=4,6$, and $8)^{8}$ and microcrystalline cellulose. ${ }^{27}$

A spectral decomposition is presented both for pyrene/calix[4]arene and pyrene microcrystals room-temperature luminescence emission (microsecond time scale) in parts a and b of Figure 4, where Voigt profiles were used. For the crystal, three Gaussian vibronic components were obtained at 426, 449, and $474 \mathrm{~nm}$ and a broad component centered at $479 \mathrm{~nm}$ with a large Lorentzian character was also found. For the inclusion complex case, three curves similar to the vibronic ones found for the crystal case but displaced toward lower wavelengths $(3-4 \mathrm{~nm})$ and slightly broader were fitted. In what concerns the excimer band, the same spectral positions and fwhm were found in both cases. Apart from this, a band peaking at $391 \mathrm{~nm}$ with $~ 50 \%$ Lorentzian character and two Lorentzians centered at 550 and $591 \mathrm{~nm}$ could also be fitted.

Room-Temperature Laser Induced Luminescence for Benzophenone Included within Silicalite Channels. Silicalite is a dealuminated analogue of ZSM-5 zeolite. The lack of substitutional aluminum results in silicalite having no catalytic or exchange properties, compared with the ZSM-5 zeolites. ${ }^{28}$ Silicalite is the only known hydrophobic form of silica and is capable of adsorbing small organic molecules up to about $6 \AA$ of kinetic diameter, even removing them from water. ${ }^{28,29}$

Silicalite as a host provides a very rigid environment for the aromatic ketone, the internal cavities being near circular zigzag channels (free-cross-section $5.4 \pm 0.2 \AA$ ) which intersect the elliptical straight channels (free-cross-section 5.7-5.8 $\AA$ × $5.1-$ 

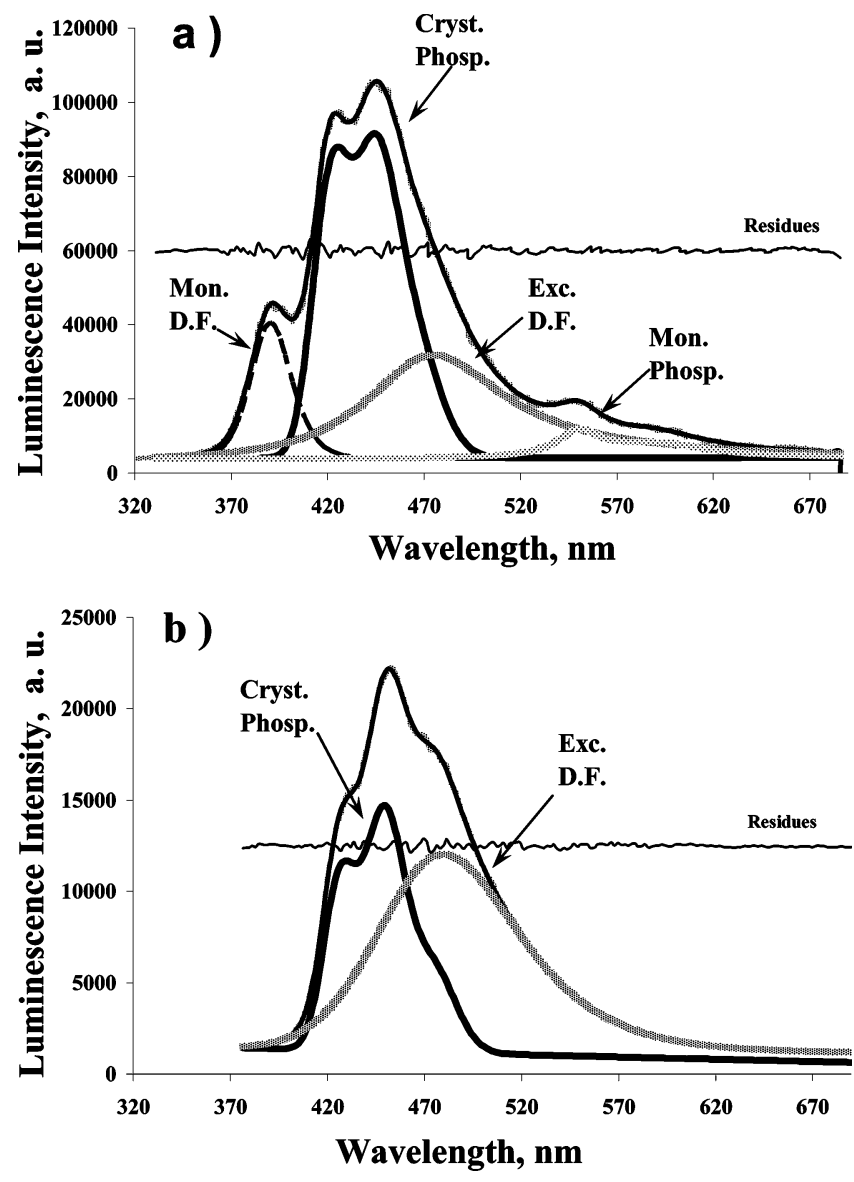

Figure 4. Luminescence spectra for pyrene adsorbed on p-tertbutylcalix[4]arene (a), and for pyrene crystals (b) excited at $337 \mathrm{~nm}$ after spectral decomposition (see text).

$5.2 \AA$ A). Both channels are defined by 10 -rings of oxygen ions. ${ }^{28}$ Therefore inclusion sites are nonhomogeneous and the phosphorescence emission spectrum of benzophenone included within silicalite channels reflects this fact: the vibronic bands are broadened as reported previously. ${ }^{30}$ Three possible locations are therefore predictable according to the silicalite structure described above: circular channels (the smaller ones), elliptical channels, and the cross-points of these two, where benzophenone may have more space available for emplacement.

It is of considerable importance to stress here that isooctane (solvent used for sample preparation) does not penetrate into the silicalite channels due to stereochemical restrictions (too big to penetrate) and also that for loadings of benzophenone greater than $100 \mu \mathrm{mol}$ per gram of silicalite, the oxygen of the air is not able to penetrate into the internal structure of silicalite and no oxygen quenching effect of benzophenone phosphorescence exists, within experimental error.

Lifetime Distribution Analysis for Pyrene/p-tert-Butylcalix[4] arene and Benzophenone /Silicalite Luminescence on the Microsecond Time Scale. To optimize the lifetime distribution analysis we recorded decay traces in several instrumental time scales, with the use of our ICCD, ranging from millisecond to sub microsecond in these cases. The experimental information obtained from this detector is of the type presented in Figure 3; i.e., from a set of time-resolved luminescence spectra and by selecting specific wavelengths, we can obtain accurate decay curves, due to the possibility of combining the use of different selected start delays, different time gate widths, and also different time steps, depending on the time range under study. In this way, the classical overloading problem of the photo- multipliers is overtaken. After these decay curves were combined, a composite decay was obtained with closely spaced points at short times and more spaced points at longer time scales (approximately equally spaced experimental data points in a $\log (t)$ scale). The methodology described previously was applied and the results obtained in this way are presented in Figures 5 and 6.

Figure 5a shows the lifetime distributions analysis for pyrene within $p$-tert-butylcalix[4]arene recovered from luminescence decays observed at $396 \mathrm{~nm}$ (delayed monomer fluorescence). Figure $5 b$ shows the lifetime distributions analysis for the same sample observed at $446 \mathrm{~nm}$ (crystal phosphorescence and delayed excimer fluorescence). Figure $5 \mathrm{c}$ shows similar data observed at $550 \mathrm{~nm}$ (pyrene monomer phosphorescence and delayed excimer fluorescence). For comparison purposes, Figure $5 \mathrm{~d}$ shows the lifetime distribution analysis obtained for pyrene microcrystals at $450 \mathrm{~nm}$ (crystal phosphorescence and delayed excimer fluorescence). The insets show the calculated curve superimposed onto the experimental data in all cases. All figures show the lifetime values at the maximum.

It is interesting to see that this lifetime distribution analysis shows large widths for the monomer delayed fluorescence and also for the pyrene microcrystals phosphorescence. In the case of the monomer delayed fluorescence this is probably related with different adsorption sites for pyrene onto the calix[4]arene host. The excimer delayed emission is much more well defined (a peak) both for pyrene/calix[4]arene and pyrene microcrystals, and this certainly reflects a more restricted possibility for the excited and nonexcited monomer to interact giving rise to an excimer formation. Most likely, fewer adsorption sites in the calixarene allow for this possibility.

Clearly all these data point to the existence of included pyrene in the pyrene/p-tert-butylcalix[4]arene samples and also to the formation of pyrene "pools" of microcrystal, as parts b and d of Figure 5 show.

In the benzophenone/silicalite case, first of all we should point that similar results were obtained both with "washed" and "nonwashed" samples of benzophenone included into silicalite, in accordance with the fact that the external surface area is negligible when compared with the internal surface area. ${ }^{31}$ Isooctane, a solvent which does not penetrate into the silicalite channels, was used for this washing procedure.

Quite surprisingly three maxima (three modal decay curve) can be visualized in the ordinates of Figure $6(\alpha$, relative abundances in the curves of the lifetime distribution analysis), and they all correspond to the luminescence of triplet excited benzophenone, i.e., phosphorescence: the first band is broad and peaks at $3.4 \mu \mathrm{s}$, an intermediate band peaking at $242 \mu \mathrm{s}$ also exists, and finally, a narrow peak in the millisecond time range $(3.1 \mathrm{~ms})$ is observed. Our interpretation is that benzophenone has three different locations in the internal structure of silicalite and that these different lifetimes reflect the significant mobility restrictions imposed by the host internal structure to the guest benzophenone. Those three different locations with increasing mobility restrictions are the intersections of the channels, the elliptical channels, and the circular channels, respectively. The intensity ratio of the three bands cannot, however, be used to have a precise evaluation of the population of the three different sites because the luminescence quantum yield of the adsorbed benzophenone varies with the adsorption site. Therefore, no linear dependence of the emission intensity vs concentration of the adsorbed probe exists.

Note that on other surfaces, e.g., in the internal hydrophilic channel structure of MCM-41 where hydroxyl groups exist, ${ }^{32}$ 

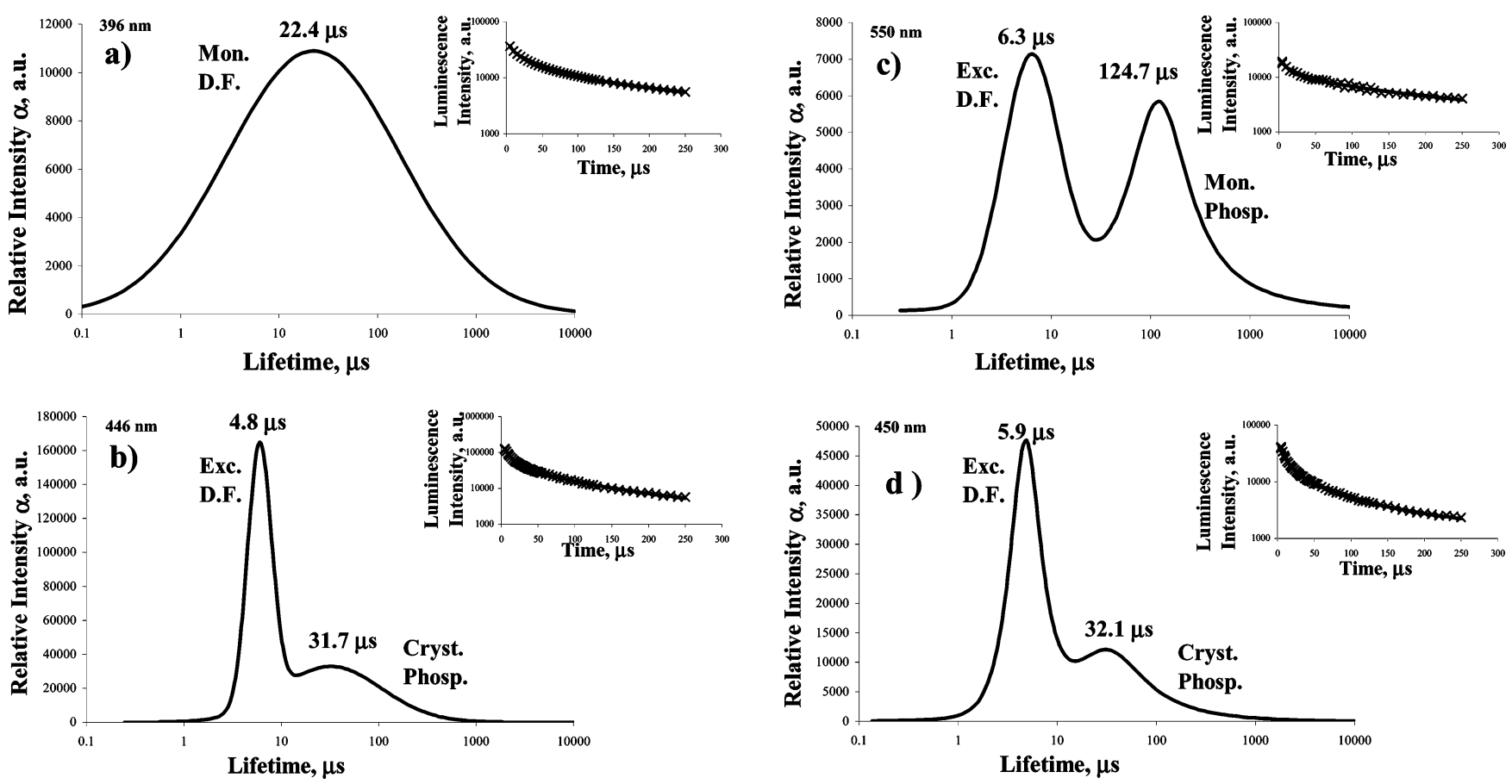

Figure 5. Lifetime distributions for pyrene on $p$-tert-butylcalix[4]arene recovered from luminescence decays observed at $396 \mathrm{~nm}$ delayed monomer fluorescence (a), $446 \mathrm{~nm}$ crystal phosphorescence and delayed excimer fluorescence (b), $550 \mathrm{~nm}$ monomer phosphorescence and delayed excimer fluorescence (c), for pyrene crystals recovered from luminescence decays observed at $450 \mathrm{~nm}$ monomer phosphorescence and delayed excimer fluorescence (d). The insets show the fitting of the recovered decay superimposed on the experimental data.

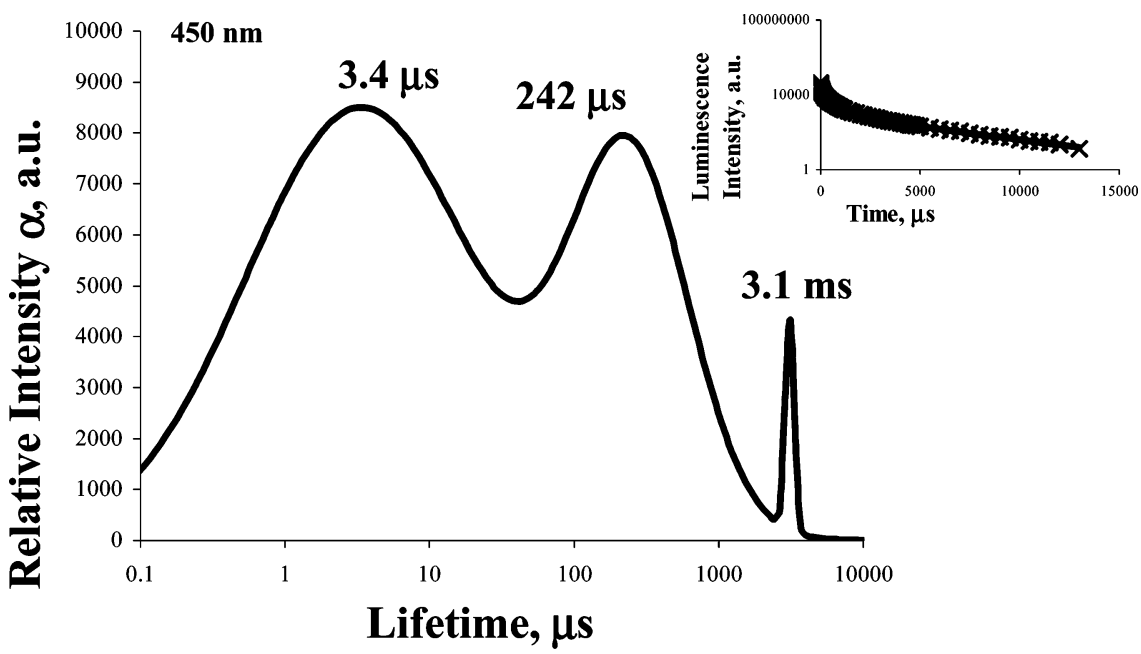

Figure 6. Lifetime distributions for benzophenone within silicalite channels recovered from luminescence decays observed at $450 \mathrm{~nm}$. The inset shows the fitting of the recovered decay to the experimental data.

another emissive form of benzophenone was detected arising from hydrogen bonded benzophenone (to those internal hydroxyl groups). In this work, where we used silicalite, which is a hydrophobic zeolite, this hydrogen bonded emissive form of benzophenone was not observed.

It is very interesting to compare the results of the use of benzophenone as a probe to study new hosts, with the ones obtained by the use of xanthone and $p$-methoxy- $\beta$-phenylpropiophenone. These three molecules are well-known probes used for surface studies in restricted media. ${ }^{33}$ The triplet-triplet absorption of xantone is known to be very sensitive to environmental polarity both in solution ${ }^{34}$ and in solid surface studies. ${ }^{33}$ In the case of $p$-methoxy- $\beta$-phenylpropiophenone the triplet lifetime is determined by intramolecular quenching by the $\beta$-phenyl ring, and the room-temperature triplet lifetime increases up to 5 orders of magnitude going from solution into restricted geometries where stretched conformations are imposed by the internal structure of the host (e.g. the silicalite case). It was believed that benzophenone was a much poorer probe than these two other ketones, because of its smaller sensitivity, either to space restrictions or environmental polarity. ${ }^{29,33}$

From other surface photochemistry studies performed using benzophenone as a probe we know now that ground state absorption spectra (obtained with the use of an integrating sphere, measuring reflectances as a function of wavelength) are highly indicative of the surface environmental polarity. ${ }^{8,27,32}$ In this report, we are showing, we believe for the first time in the literature, that a carefully study of the decay curve of benzophenone in a large time scale obtained with the use of a gated detector, followed by a lifetime distribution analysis with this new methodology can provide very accurate information relative to the existence of different adsorption sites in a specific solid powdered host. 


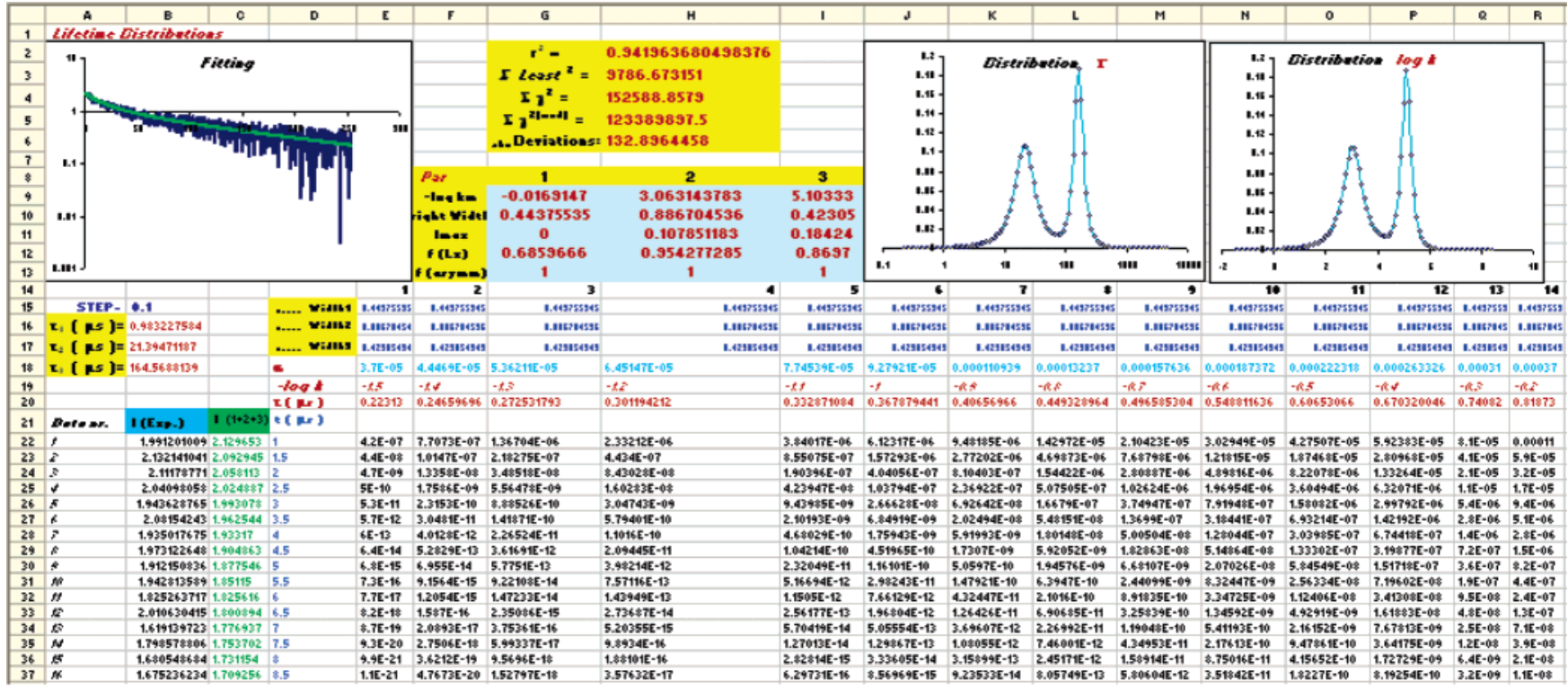

Figure 7. Display of the worksheet showing the most important fields described above.

\section{Conclusions}

The new methodology is presented here for lifetime distribution analysis, which, conjugated with the knowledge of laser induced time-resolved luminescence spectra, proved to be a very powerful tool for the understanding of the luminescence emission of guest molecules onto powdered solid substrates. In the pyrene/calix[4]arene case delayed fluorescence emission from monomers and excimers exist, as well as monomers phosphorescence and crystals phosphorescence. In the benzophenone/silicalite case, three different phosphorescence emissions from included benzophenone were detected, showing how sensitive benzophenone is as a probe for revealing different adsorption sites in the internal structure of the host.

Acknowledgment. T.J.F.B. thanks FCT for a Doctoral fellowship SFRH/BD/8143/2002, and I.F.M. thanks FSE for financial support.

Supporting Information Available: Figures for several tests made with and without added noise showing the simulated and the fitted distributions. This material is available free of charge via the Internet at http://pubs.acs.org.

\section{Appendix 1. Detailed Description of the Worksheet}

Figure 7 shows a display of the worksheet with the following points.

-A graphic with the decay curves ([A2: E13]), the experimental (black) and the fitted one (red).

-Two graphics with the distribution in terms of $\ln (k)$ ([N2: Q13]) and in terms of lifetime, ([J2: M13]).

-The amounts to be minimized-least squares [H3], $\chi^{2}$ [H4], modified $\chi^{2}[\mathrm{H} 5]$, and the mean value of maximum and minimum absolute deviations [H6]. All of them were multiplied by 1000 to increase the precision of the fitting. Also the correlation coefficient [H2], computed by Excel is shown.

- The table of parameters to be fitted by the Solver for each of the three fitted distributions ([G9: I13]): the maximum position of the distribution in terms of $\ln (k)$ [G9: I9]; width of the right side of the distribution [G10: I10]; the height of the distribution [G11: I11]; the Lorentzian fraction of the distribution [G12: I12]; the asymmetry factor of the distribution [G13: I13].
-The step ([B15]) that defines the range of the distributions in terms of $-\ln (k)$ [E19: GV19].

-The maximum position of the fitted distributions in terms of lifetimes [B16: B18] (defined in terms of $-\ln (k)$ ).

-A row ([E19: GV19]) with the abscissas (100, in this example) of the fitted distributions in term of $-\ln (k)$ generated with the step defined in [B15].

-A row ([E20: GV20]) with the abscissas of the fitted distributions in term of equivalent lifetimes [E19: GV19].

-Effective width ([E15: GV17]) of distributions 1, 2, and 3 taking into account the asymmetry by means of an IF condition.

-Enumeration ([A22: A221]) of the experimental data points (200, in this case).

-A row ([E18: GV18]) with the weight $\alpha$ terms of each $\ln (k)$ in the distribution (sum of the 3 fitted distributions).

-Rows [E22: GV221] containing exponential terms (exp($t k)$ ), for each $\ln (k)$, multiplied by $\alpha$.

-A column with the experimental times of the decay measurements ([D22: D221]).

-A column with the experimental decay intensities ([B22: B221]).

-A column with the fitted decay intensities ([C22: C221]): it is the result of the numerical calculation of the integral in eq 6. Each cell $[\mathrm{Cx}]$ is the sum of the entire row $[\mathrm{Ex}: \mathrm{CVx}]$.

-A column with the square deviations ([GW22: GW222]) between the experimental and fitted intensities for each instant $t$. Its sum, multiplied by 1000 , is in cell $\mathrm{H} 3$ as referred above.

-A column with the relative square deviations [GX22: GX222] for each $t$. Its sum, multiplied by 1000 , is in cell H4.

-A column with the square deviations [GY22: GY222] divided by the square experimental intensity for each $t$. Its sum, multiplied by 1000 , is in cell $\mathrm{H} 5$.

-A column with the absolute deviations [GZ22: GZ222] between the experimental and fitted intensities for each t. The maximum and the minimum of this column are, respectively, in cells [GZ18] and [GZ19]. Their mean value is in cell H6.

Formulas in the cells:

-[H2] = CORREL(B22:B221,C22:C221)

-[H3] = SUM(GW22: GW222)*1000

-[H4] = SUM (GX22: GX222)*1000

-[H5] = SUM (GY 22: GY 222)*1000

$\cdot[\mathrm{H} 6]=(\mathrm{GZ} 18+\mathrm{GZ} 19) / 2 * 1000$ 
$\bullet[\mathrm{A} 16: \mathrm{A} 18]=1 /(\operatorname{EXP}(-\mathrm{G} 9)) ;=1 /(\operatorname{EXP}(-\mathrm{H} 9)) ;=1 /(\mathrm{EXP}-$ $(-\mathrm{I} 9))$

-[E19: GV19] =E19 (initial value of $-\ln (k))$; a19= $(\mathrm{a}-1)$ $19+\$ B \$ 15(\mathrm{a}=\mathrm{F}$ to $\mathrm{GV})$

-[E20: GV20] $=1 /(\operatorname{EXP}(-\mathrm{a} 19))(\mathrm{a}=\mathrm{E}$ to $\mathrm{GV})$.

-[E15: GV17] $=\mathrm{IF}(\mathrm{a} \$ 19<\$ \mathrm{~b} \$ 9, \$ \mathrm{~b} \$ 10, \$ \mathrm{~b} \$ 10 * \$ \mathrm{~b} \$ 13)$-with

$\mathrm{a}=\mathrm{E}$ to $\mathrm{GV}$ and $\mathrm{b}=\mathrm{G}, \mathrm{H}$ and $\mathrm{I}$ for, respectively, the 1st, the 2nd, and the 3rd distributions.

-[E18: $\mathrm{GV} 18]=(\$ \mathrm{G} \$ 11 /(1+\$ \mathrm{G} \$ 12 *(\mathrm{a} \$ 19-\$ \mathrm{G} \$ 9) \wedge 2 * 4 /$ $\mathrm{E} \$ 15 \wedge 2) * \operatorname{EXP}(-(1-\$ \mathrm{G} \$ 12) *(\mathrm{a} \$ 19-\$ \mathrm{G} \$ 9) \wedge 2 * \mathrm{LN}(2) * 4 /$ $\mathrm{E} \$ 15 \wedge 2))+(\$ H \$ 11 /(1+\$ H \$ 12 *(\mathrm{a} \$ 19-\$ H \$ 9) \wedge 2 * 4 / \mathrm{E} \$ 16 \wedge 2)-$ $* \operatorname{EXP}(-(1-\$ H \$ 12) *(a \$ 19-\$ H \$ 9) \wedge 2 * L N(2) * 4 / E \$ 16 \wedge 2))+(\$ I \$ 11 /$ $(1+\$ \mathrm{I} \$ 12 *(\mathrm{a} \$ 19-\$ \mathrm{I} \$ 9) \wedge 2 * 4 / \mathrm{E} \$ 17 \wedge 2) * \mathrm{EXP}(-(1-\$ \mathrm{I} \$ 12) *(\mathrm{a} \$ 19-$ $\$ \mathrm{II} 9) \wedge 2 * \mathrm{LN}(2) * 4 / \mathrm{E} \$ 17 \wedge 2))(\mathrm{a}=\mathrm{E}$ to $\mathrm{GV})$.

-[E22: GV221] $=\mathrm{a} \$ 18 * \operatorname{EXP}(-\$ D x * \operatorname{EXP}(-\mathrm{a} \$ 19))$ with $\mathrm{a}=\mathrm{E}$ to $\mathrm{GV}$ and $x=22$ to 221 .

-[C22: $\mathrm{C} 221]=\mathrm{SUM}(\mathrm{Ex}: \mathrm{GV} x)(\mathrm{x}=22$ to 221$)$.

-[GW22: GW221] $=(\mathrm{Bx}-\mathrm{Cx}) \wedge 2(\mathrm{x}=22$ to 221$)$.

-[GX22: GX221] = GWx/Bx (x=22 to 221).

-[GY22: GY221] = GWx/(Bx^2) $(\mathrm{x}=22$ to 221$)$.

-[GZ22: GZ221] = ABS(Bx-Cx $)(x=22$ to 221$)$.

-[GZ18] =MAX $(\mathrm{GZ} \$ 19: \mathrm{GZ} \$ 221)$

•[GZ19] $=\mathrm{MIN}(\mathrm{GZ22}: \mathrm{GZ221})$

\section{Appendix 2 - Optimization protocol}

For the solver options:

-Maximum time and number of iterations

-Maximum precision, tolerance and convergence

-Quadratic estimates

-Forward derivatives

-Newton search

Set the target cell to the value of " 0 ", subject to the constraints:

-\$G\$10:\$I $\$ 10>=0.01$

-\$G\$10:\$I\$11>=0

-\$G\$12:\$I\$12<= 1

-\$G\$12:\$I $\$ 12>0$

-\$G\$13:\$I $\$ 13>=0.01$

For the initial guess the following steps were pursued:

(1) Choice of three $\ln (\bar{k})$ so that the corresponding lifetimes $(\bar{\tau}=1 / \bar{k})$ fall inside the time range of the decay curve. For instance, for a decay curve ranging from $0.2 \mu \mathrm{s}$ to $5 \mu \mathrm{s}$, we chose, invariably, $\ln (\bar{k})=-1,0$ and $1(\bar{\tau}=2.7,1$, and 0.367 $\mu \mathrm{s})$.

(2) All the widths with the same initial value $(0.5$, for instance) and a step (cell B15) of 0.1 .

(3) All the Lorentzian weights at 0 (pure Gaussian distributions).

(4) All the asymmetry factors equal to 1 (symmetrical distributions)

(5) All the intensities with the same value. The value is manually chosen so the calculated decay is the closest possible to the experimental one.

(6) At this point, value in cell E22 should be of the order of $10^{-7}$. If it is smaller (larger), the first value of $-\ln (\bar{k})$ [cell E19] must be increased (decreased) to bring that value to $10^{-7}$. Next, the value in the last calculated column, first row (cell GV22) shall be inspected. Again, if it is lower (larger) than $10^{-7}$, the step (cell B15) should be decreased (increased).

(7) The solver is then run taking as target cell the least squares [cell H3] and choosing as range to be fitted cells containing the maximum positions of the distribution in terms of $\ln (k)$ [G9: I9], the width of the right side of the distribution [G10: I10] and the height of the distribution [G11: I11]. Lorentzian fraction and asymmetries are kept constant.

(8) Once it converges, step 6 should be repeated. Then, step 7 is repeated but using the $\chi^{2}$ [cell H4] as target value in the solver. An alternation between the least squares [cell H3], the $\chi^{2}$ [cell H4], and the modified $\chi^{2}$ [cell H5] should improve the overall fitting.

(9) The solver is then run taking as target cell the least squares [cell H3] and choosing as range to be fitted all the cells (containing the maximum positions of the distribution, in terms of $\ln (k)$ [G9: I9], the width of the right side of the distribution [G10: I10], the height of the distribution [G11: I11] and Lorentzian fraction [G12: I12]. Asymmetries are kept constant.

(10) Repeat step 8 .

(11) The solver is then run taking as target cell the least squares [cell H3] and choosing as range to be fitted all the cells [G9: I12].

(12) Repeat step 8.

Notes

-All the cells must have a high number of decimal places. The number category in the cells format should be general.

-Each time the solver is run, it should be allowed to finish the optimization, although it can be suspended with the "Esc" key in order to verify the fitting status.

-Whenever a distribution systematically goes out of the fitting range, that specific distribution may be due to an experimental artifact, like noise or inconsistent data, and should not be considered in the final results.

-If the distributions become increasingly thinner or asymmetric, the optimization should be stopped and that trend should be corrected by not choosing as range to be fitted the cells containing the width of the right side of the distribution [G10: I10], or the asymmetry [G13: I13] of the distribution. Asymmetry is a fine-tune and should only be run at the end of the optimization protocol.

-Whenever two distributions are too close together, they might be only one wider or asymmetric distribution, and the solver should be allowed to try to optimize only one distribution, and determine the best overall fitting.

-The best final criterion for optimization of parameters is usually the least squares, but in cases where the difference between the initial and the final decay values does not exceed 1 order of magnitude, the modified $\chi^{2}$ can also be used as final target cell.

-Whenever the results are poor, one can always modify the worksheet, increasing the number of columns, and that will surely improve the fitting.

\section{References and Notes}

(1) (a) Botelho do Rego, A. M.; Vieira Ferreira, L. F. In Handbook of Surfaces and Interfaces of Materials; Nalwa, H. S., Ed.; Academic Press: New York, 2001; Vol. 2, Chapter 7, pp 275-313. (b) Vieira Ferreira, L. F.; Branco, T. J. F.; Botelho do Rego, A. M. Chem. Phys. Chem. 2004, 5, $1-7$.

(2) Scaiano, J. C.; Garcia, H. Acc. Chem. Res. 1999, 32, 783-793.

(3) Turro, N. J. Acc. Chem. Res. 2000, 33, 637-646.

(4) Hashimoto, S. J. Photochem. Photobiol. C: Photochem. Rev. 2003, 4, 19-49.

(5) Thomas, J. K. Chem. Rev. 1993, 93, 301-320.

(6) (a) Sikorski, M.; Jasiewicz, B.; Wojciak, A.; Khmelinskii, I. V.; Bourdelande, J. L.; Sikorska, E. Trends Phys. Chem. 2003, 9, 103-117. (b) Sikorski, M.; Mir, M.; Wilkinson, F. Chem. Commun. 1997, 395-396. (c) Sikorski, M.; Sikorska, E.; Khmelinskii, I. V.; Moreno, R. G.; Bourdelande, J. L.; Siemiarczuk, A. Photochem. Photobiol. Sci. 2002, 1, 715-720.

(7) Vieira Ferreira, L. F.; Vieira Ferreira, M. R.; Da Silva, J. P.; Ferreira Machado, I.; Oliveira, A. S.; Prata, J. V. Photochem. Photobiol. Sci, 2003, 2, 1002-1010. 
(8) Vieira Ferreira, L. F.; Vieira Ferreira, M. R.; Oliveira, A. S.; Branco, T. J. F.; Prata, J. V.; Moreira, J. C. Phys. Chem. Chem. Phys. 2002, 4 204-210. 188.

(9) Oelkrug, D.; Kortum, G. Z. Phys. Chem. N. F. 1968, 58, 181-

(10) (a) Vieira Ferreira, L. F.; Freixo, M. R.; Garcia, A. R.; Wilkinson, F. J. Chem. Soc., Faraday Trans. 1992, 88, 15-22. (b) Vieira Ferreira, L. F.; Garcia, A. R.; Freixo, M. R.; Costa, S. M. B. J. Chem. Soc. Faraday Trans. 1993, 89, 1937-1944.

(11) Vieira Ferreira, L. F.; Ferreira Machado, I.; Oliveira, A. S.; Vieira Ferreira, M. R.; Da Silva, J. P.; Moreira, J. C. J. Phys. Chem. B 2002, 106, 12584-12593.

(12) (a) Schemiarczuk, A.; Ware, W. R. J. Phys. Chem. 1987, 91, 36773682. (b) Schemiarczuk, A.; Ware, W. R.; Chem. Phys. Lett. 1989, 160, 285-290. (c) Schemiarczuk, A.; Wagner, B.; Ware, W. R. J. Phys. Chem. 1990, 94, 1661-1666. (d) Wagner, B.; Ware, W. R. J. Phys. Chem. 1990, 94, 3489-3494. (e) Ware, W. R. In Photochemistry in Organized and Constrained Media; Ramamurty, V., Ed.; VCH Publishers: New York, 1991; Chapter 13, pp 563-602. (f) Liu, Y. S.; P.; Ware, W. R. J. Phys. Chem. 1993, 97, 5980-5986. (g) Liu, Y. S.; de Mayo, P.; Ware, W. R. J. Phys. Chem. 1993, 97, 5987-5994. (h) Liu, Y. S.; de Mayo, P.; Ware, W. R. J. Phys. Chem. 1993, 97, 5995-6001.

(13) (a) Albery, W. J.; Bartlett, P. N.; Wilde, C. P.; Darwent, J. R. J. Am. Chem. Soc. 1985, 107, 1854-1862. (b) Scott, K. F. J. Chem. Soc., Faraday Trans. I 1980, 76, 2065-2072.

(14) (a) Worrall, D. R.; Kirkpatrick, I.; Williams, L. Photochem. Photobiol. Sci, 2004, 3, 63-70. (b) Levy P. Theorie de l'addition des variables aleatoires; Guthier Villars: Paris, France, 1937.

(15) Bright, F. V.; Catena, G. C.; Huang, J. J. Am. Chem. Soc. 1990, 112, 1343-1346.

(16) Kranansky, R.; Koike, K.; Thomas, J. K. J. Phys. Chem. 1990, 94, $4521-4528$

(17) (a) Barra, M.; Scaiano, J. C. Photochem. Photobiol. 1995, 62, 6064. (b) Barra, M.; Agha, K. A. J. Photochem. Photobiol. A: Chem. 1997, 109, 293-298. (c) Barra, M.; Agha, K. A. Supramol. Chem. 1998, 10, $91-$ 95 .

(18) (a) Gutshe, C. D. Calixarenes; Royal Society of Chemistry: Cambridge, U.K., 1989. (b) Alam, I.; Gutsche, C. D. J. Org. Chem. 1990, 55, 4487-4489. (c) Gutsche, C. D.; Alam, I. Tetrahedron 1988, 44, 46894694.
(19) Rudkevich, D. M. Bull. Chem. Soc. Jpn. 2002, 75, 393-413.

(20) Anpo, M.; Nishiguchi, H.; Fujii, T. Res. Chem. Interm. 1990, 13 73-102. (b) Negishi N.; Fujii, T.; Anpo, M. Heterogen. Chem. Rev. 1994, $1,231-141$.

(21) (a) De Mayo, P.; Natarajan, L. V.; Ware, W. R. ACS Symp. Ser. 1985, 278, 1-19. (b) Bauer, R. K.; De Mayo, P.; Ware, W. R.; Wu, K. C. J. Phys. Chem. 1982, 86, 3781-3789.

(22) Li, L.; Zhang, Z.; Long, W.; Tong, A. Spectrochim. Acta A 2001, $56,385-393$.

(23) Castillo, A. S.; Caretero, A. S.; Costa Fernandez, J. M.; Jin, W. J.; Gutierrez, A. F. Anal. Chim. Acta 2004, 516, 213-220.

(24) (a) Lochmullar, C. H.; Wenzel, T. J. J. Phys. Chem. 1990, 94, 4230-4235. (b) Gijzeman, O. L. J.; Langelaar, J.; Van Voorst, J. D. W. Chem. Phys. Lett. 1970, 5, 269-272. (c) Stevens, B. Spectrochim. Acta 1962, 18, 439-438.

(25) Sal'nikov, A. N.; Spivak, A. V.; Mel'nikov, G. V. Russ. Phys. J. 2002, 45, 570-572.

(26) Tozuka, Y.; Yonemochi, E.; Ogushi, T.; Yamamoto, K. J. Colloid Interface Sci. 1998, 205, 510-515.

(27) Vieira Ferreira, L. F.; Netto-Ferreira, J. C.; Khmelinskii, I.; Garcia,

A. R.; Costa, S. M. B. Langmuir 1995, 11, 231-236.

(28) (a) Flaningen, E. C.; Bennet, J. M.; Grose, R. W.; Patton, R. L.; Kirchner, R. M.; Smith, J. V. Nature 1978, 271, 512-516. (b) ShultzSibbel, G. M. W.; Gjerde, D. T.; Chriswell, C. D.; Fritz, J. S.; Colleman, W. E. Talanta 1982, 29, 447-452. (c) Bibby, D. M.; Millestone, N. B.; Aldridge, L. P. Nature 1979, 280, 664-665.

(29) (a) Vieira Ferreira, L. F.; Oliveira, A. S.; Netto-Ferreira, J. C. In Fluorescence Microscopy and Fluorescence Probes 3; Kotyk, A. Ed.; Espero Publishing: Prague, 1999; pp 199-208. (b) Vieira Ferreira, L. F.; NettoFerreira, J. C.; Costa, S. M. B. Spectrochim. Acta A 1995, 51, 1385-1388. (30) Vieira Ferreira, L. F.; Vieira Ferreira, M. R.; Oliveira, A. S.; Moreira, J. C. J. Photochem. Photobiol., A: Chem. 2002, 153, 11-18.

(31) Leigh, W. J.; Johnson, L. J. In Handbook of Organic Photochemistry; Scaiano, J. C., Ed.; CRC Press: Boca Raton, FL, 1989; Vol. 2, Chapter 22, pp 401-422.

(32) Da Silva J. P.; Ferreira Machado, I.; Lourenço, J. P.; Vieira Ferreira, L. F. Microporous Mesoporous Mater 2005, in press.

(33) Scaiano, J. C.; Kaila, M.; Corrent, S. J. Phys. Chem. B 1997, 101, 8564-8568.

(34) Scaiano, J. C. J. Am. Chem. Soc. 1980, 102, 7747-7753 